ARTICLE

DOI: $10.1038 / \mathrm{s} 41467-017-02778-5$

\title{
DNA-assisted swarm control in a biomolecular motor system
}

\author{
Jakia Jannat Keya ${ }^{1}$, Ryuhei Suzuki ${ }^{1}$, Arif Md. Rashedul Kabir², Daisuke Inoue ${ }^{2}$, Hiroyuki Asanuma ${ }^{3}$, Kazuki Sada ${ }^{1,2}$, \\ Henry Hess (1) ${ }^{4}$, Akinori Kuzuya (1D ${ }^{5}$ \& Akira Kakugo $1,2,4$
}

In nature, swarming behavior has evolved repeatedly among motile organisms because it confers a variety of beneficial emergent properties. These include improved information gathering, protection from predators, and resource utilization. Some organisms, e.g., locusts, switch between solitary and swarm behavior in response to external stimuli. Aspects of swarming behavior have been demonstrated for motile supramolecular systems composed of biomolecular motors and cytoskeletal filaments, where cross-linkers induce large scale organization. The capabilities of such supramolecular systems may be further extended if the swarming behavior can be programmed and controlled. Here, we demonstrate that the swarming of DNA-functionalized microtubules (MTs) propelled by surface-adhered kinesin motors can be programmed and reversibly regulated by DNA signals. Emergent swarm behavior, such as translational and circular motion, can be selected by tuning the MT stiffness. Photoresponsive DNA containing azobenzene groups enables switching between solitary and swarm behavior in response to stimulation with visible or ultraviolet light.

\footnotetext{
${ }^{1}$ Graduate School of Chemical Sciences and Engineering, Hokkaido University, Sapporo, 060-0810, Japan. ${ }^{2}$ Faculty of Science, Hokkaido University, Sapporo, 060-0810, Japan. ${ }^{3}$ Graduate School of Engineering, Nagoya University, Osaka, 564-8680, Japan. ${ }^{4}$ Department of Biomedical Engineering, Columbia University, 1210 Amsterdam Avenue, New York, NY, 10027, USA. ${ }^{5}$ Department of Chemistry and Materials Engineering, Kansai University, Osaka, 5648680, Japan. Correspondence and requests for materials should be addressed to A.K. (email: kuzuya@kansai-u.ac.jp)

or to A.K. (email: kakugo@sci.hokudai.ac.jp)
} 
$\mathrm{N}$ ature assembles its wide variety of structures through selfassembly and self-organization where local interactions among the individual components play pivotal roles $^{1}$. A striking example is the swarming of living organisms in which fascinating, large scale and complex structures emerge from local interactions among the individuals rather than through control by a leader ${ }^{2}$. Fish schools, ant colonies, and bird flocks are typical examples of swarming observed in nature. Swarming grants several advantages to the organism, including parallelism, robustness, and flexibility; all of these are unachievable by a single entity ${ }^{3,4}$. Inspired by the attractive features of swarming organisms, researchers working in the fields of robotics and nanotechnology have been investigating the swarming of self-propelled mechanical devices ${ }^{5-7}$ and chemically powered self-propelled particles $^{8-10}$, respectively. A major challenge in swarm robotics is the construction of large numbers of individual robots capable of programmable self-assembly ${ }^{11}$

This challenge may be addressed by the creation of molecular robots. Molecular robots are molecular systems composed of all of the three essential components of robots: sensors, information processors, and actuators ${ }^{12-14}$. The systems integrating biomolecular motors and their corresponding cytoskeletal filaments, such as myosin-actin, dynein-MT, or kinesin-MT systems, can provide a large number of self-propelled molecular entities ${ }^{15,16}$. Recent studies have demonstrated some aspects of swarming behavior by controlling the mutual interactions of the propelled filaments using associated proteins ${ }^{17}$, depletion agents ${ }^{18}$, crowding effects $^{19-21}$, or ligand-receptor-based crosslinking ${ }^{22-25}$. These interactions lead to the emergence of fascinating swarm patterns, such as bundles, spools, vortex lattices ${ }^{26,27}$, as well as circular or polar patterns ${ }^{28}$. However, programmability of the interactions, critical to the exploration of swarm behavior at the macroscale ${ }^{11}$, has not yet been achieved in these systems. Therefore, the scientific questions are if a linker exists that combines sufficient interaction strength, selectivity, reversibility, and controllability to enable these systems to respond with distinct behaviors, and if that linker can provide sensing and information processing capabilities to these systems composed of cytoskeletal filaments propelled by biomolecular motors.

We demonstrate, in this study, that DNA can be employed as a universal interface to control the swarming of MTs in a programmable manner. The unique features of DNA as a storage device of genetic information, i.e., strict sequence-selectivity managed by complementary base-pairing and uniform righthanded double helical structure, together with recent progress in chemical DNA synthesis, have made DNA a versatile tool for molecular computing ${ }^{29,30}$, and a building block for the construction of nanostructures ${ }^{31,32}$. DNA has already been used in combination with biomolecular motor systems, either acting as a highly specific glue to assemble motors into multimers ${ }^{33,34}$, or to connect motors to DNA origami scaffolds ${ }^{35,36}$, or being conjugated to MTs for the purpose of cargo loading/unloading ${ }^{37-45}$. Here, we report the control of swarming of kinesin-propelled MTs (actuators) by tethering single-stranded DNA to the MTs and programming the interactions among MTs using DNA crosslinkers as input signals (information processing). We also regulated the swarming mode of the MTs, such as swarming with translational or circular motion, by tuning the physical properties of the MTs. By introducing a photoresponsive residue, azobenzene, into the DNA strands as a sensor, the swarming of the MTs was further controlled by photoirradiation in a reversible fashion.

\section{Results}

Design of swarming of MTs controlled by DNA. We prepared the individual swarm units by conjugating MTs with single- stranded DNA, at a labeling ratio of about one strand per tubulin dimer, through a copper-free click reaction. Either the DNA or the MTs were labeled with a fluorescent dye in order to allow monitoring of the MTs under a fluorescence microscope (Fig. 1a). The MTs are cylindrical objects with an outer diameter of $25 \mathrm{~nm}$ and lengths between 2 and $10 \mu \mathrm{m}$. Based on the results of melting temperature $\left(T_{\mathrm{m}}\right)$ simulation, the base number in a DNA strand was fixed to 16 to obtain a $T_{\mathrm{m}}$ above the working temperature of the biomolecular motor system $\left(25^{\circ} \mathrm{C}\right)$. The DNA-conjugated MTs were propelled by surface-adhered kinesins using the chemical energy of adenosine triphosphate (ATP $)^{46}$. Smooth gliding of the DNA-conjugated MTs confirmed that the DNA conjugation does not hinder the interaction of MTs with kinesins (Supplementary Figs. 1 and 2 and Supplementary Tables 1 and 2).

Demonstration of reversible swarming of MTs triggered by DNA. Two DNA strands, $\mathrm{T}_{16}$ and (TTG) 5 , were labeled with the fluorescent dyes TAMRA (red) and FAM (green), respectively. These strands, termed receptor DNA ( $r$-DNA), were conjugated to MTs, yielding two types of MTs distinguishable by their fluorescence (Fig. $1 \mathrm{~b}$ and Supplementary Table 3). We placed equal numbers of red ( $\mathrm{T}_{16}$-labeled) and green ((TTG) $)_{5}$-labeled) MTs on a kinesin-coated substrate (Fig. 1c) at a combined density of $50,000 \mathrm{~mm}^{-2}$. In the presence of ATP, the MTs moved without any interaction with each other (Fig. 1d and Supplementary Movie 1). Linker DNA (l-DNA1) was designed to be partially complementary to the $r$-DNAs, so that it can cross-link them (Fig. 1e and Supplementary Table 3). Association of the MTs was then initiated by the introduction of $l$-DNA 1 as the input signal (Fig. 1e-g). While gliding, the red and green MTs came close to each other, associated into swarms (appearing yellow in the merged images), and continued moving (Fig. 1g). The size of the swarms of MTs increased by mergers of swarms, decreasing the density of swarms over time (Fig. $1 \mathrm{~g}$ and Supplementary Movie 1). Despite the increase in size, the swarms of MTs exhibited translational motion with a velocity $(0.51 \pm 0.02 \mu \mathrm{m} \mathrm{s}$ ${ }^{-1}$ ) close to that of individual MTs $\left(0.60 \pm 0.05 \mu \mathrm{m} \mathrm{s}^{-1}\right)$. We counted the number of individual MTs at different time points and characterized the swarming of MTs by calculating the association ratio defined as the fraction of the number of initial MTs incorporated into the swarms (see Supplementary Methods). The association ratio increased with time and reached a plateau ( 90-95\%) within 60 min after the addition of $l$-DNA1 (Fig. 1i). In the absence of kinesin, freely diffusing MTs formed unstructured aggregates whereas immobile MTs did not interact in the presence of kinesin and absence of ATP (Supplementary Fig. 3).

Dissociation of the swarms into individual MTs was then demonstrated by introducing dissociation DNA ( $d$-DNA), which was designed to extract l-DNA1 through a DNA strand exchange reaction (Figs. 1e, f and Supplementary Table 3$)^{47}$. The yellow-colored swarms of MTs dissociated into red and green MTs after the introduction of $d$-DNA (Figs. 1h, i). By counting the individual MTs present after dissociation (Fig. $1 \mathrm{i}$ and Supplementary Movie 1), it was estimated that a swarm is composed of $\sim 100$ individual MTs.

DNA-based logic gates to control the swarming of MTs. Based on the utility of DNA as an operator for molecular computing ${ }^{30}$, we aimed to demonstrate different logic operations where swarming was the output controlled by DNA inputs (Supplementary Table 4). A YES logic gate was realized by using $l$-DNA1 as input 1 , and the swarming of red and green MTs as the output 1 (Fig. 2). The AND logic gate was demonstrated by designing two different linker DNA signals as $l$-DNA2 and 
a
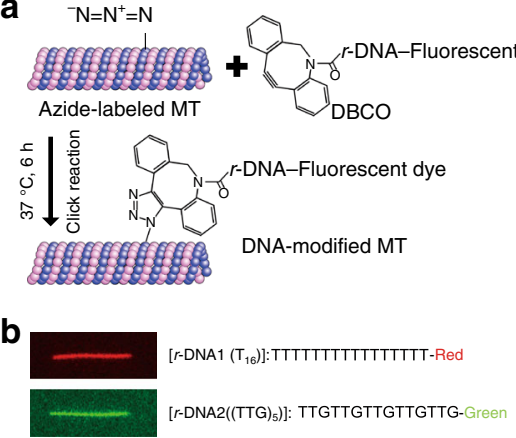

C

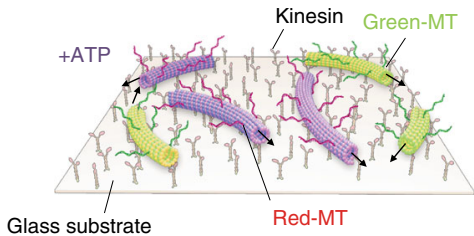

e

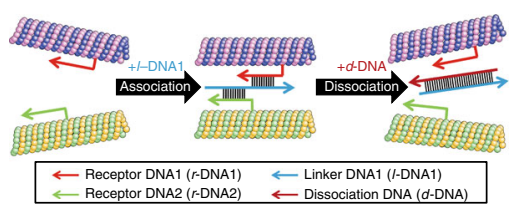

$\mathbf{f}$

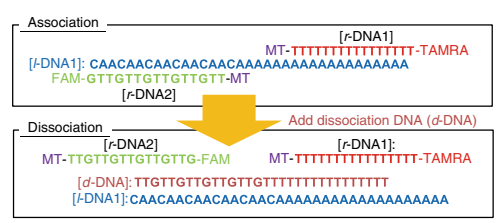

i

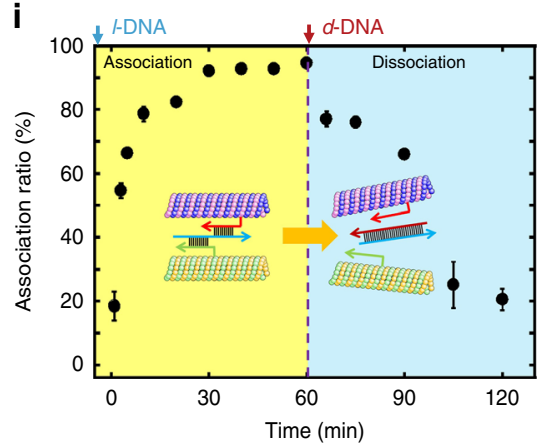

Fig. 1 Preparation of MTs and control of their swarming. a Conjugation of $r$-DNA to azide-functionalized MTs by a click reaction. $\mathbf{b}$ TAMRA (red) and FAM (green) labeled DNA-conjugated MTs with sequences of $\mathrm{T}_{16}$ ( $r$-DNA1) and (TTG) 5 ( $r$-DNA2), respectively. Scale bar: $5 \mu$ m. c Schematic of red and green MTs gliding on kinesins. $\mathbf{d}$ Time-lapse images showing motility of rigid MTs with lengths of $5 \pm 2$ and $5 \pm 3 \mu \mathrm{m}$ (average \pm s.d.) conjugated to $\mathrm{T}_{16}$ and (TTG) ${ }_{5}$, respectively. Scale bar: $10 \mu \mathrm{m}$. e Schematic of the association of red and green MTs by I-DNA1 and their dissociation by $d$-DNA via extraction of I-DNA1 through a DNA strand exchange reaction. $\mathbf{f}$ Association and dissociation of MTs by DNA base pair interaction. $\mathbf{g}$ Time-lapse images showing swarming of MTs. $\mathbf{h}$ Dissociation of swarms of MTs. Scale bar: $20 \mu \mathrm{m}$ ( $\mathbf{g}$ and $\mathbf{h})$. i Change of association ratio over time after introduction of I-DNA1 (0.6 $\mu$ M) and $d$-DNA (0.6 $\mu$ M). The concentration of the red and green MTs was $0.6 \mu \mathrm{M}$ each, and the kinesin concentration was $0.3 \mu \mathrm{M}$. Error bar: standard error (s.e.m.)

l-DNA3, which are partially complementary to $r$-DNA1 and $r$-DNA2, respectively, and also to each other. The OR logic gate was implemented by conjugating pairs of $r$-DNA to the MTs $(r$ DNA1 and $r$-DNA3 labeled with TAMRA; $r$-DNA2 and $r$-DNA4 labeled with FAM), and using $l$-DNA1 (complementary to $r$ DNA1 and $r$-DNA2) and $l$-DNA4 (complementary to $r$-DNA3 and $r$-DNA4) as the two input signals. Association ratios of $85-100 \%$ were obtained for all of the systems expecting the output representing 1 (Fig. 2), which are significantly higher than those for the outputs representing $0(<5 \%)$.

Swarming modes regulated by the physical properties of MTs. We have previously found that the swarming mode of MTs is controlled by the rigidity and length of the MTs ${ }^{48,49}$. We prepared MTs with lower rigidity by polymerizing MTs with guanosine triphosphate (GTP) guanylyl- $(\alpha, \beta)$-methylenedisphosphonate (GMPCPP) used in the experiments described above $^{50}$. The flexible GTP-MTs were then conjugated with $r$ DNA1 and $r$-DNA2. While gliding on kinesins, the flexible MTs moved in more curved paths compared to the rigid MTs (Fig. 3a, b, Supplementary Movie 2). The path persistence length, $L_{\mathrm{p}}$, of the flexible GTP-MTs was $245 \pm 32 \mu \mathrm{m}$ (Supplementary Fig. 4), while that of the rigid GMPCPP-MTs was $582 \pm 97 \mu \mathrm{m}$, reflecting a more than two-fold difference in rigidity. Unlike the rigid MTs, the flexible MTs exhibited swarming with circular motion upon the input of $l$-DNA1 (Fig. 3c, d, and Supplementary Movie 2). The swarms with circular motion can also be dissociated into single MTs by introducing the input $d$-DNA signal (Fig. $3 \mathrm{e}, \mathrm{f}$, and
Supplementary Movie 2). Single MTs retained their gliding motion on the kinesin-coated substrate.

Orthogonal control of swarming of MTs. The selective hybridization property of DNA allowed us to design a system exhibiting controlled swarming of flexible and rigid MTs without any crosstalk. We conjugated two types of MTs, which differed in body length and rigidity, with two different DNA logic gates (Fig. 4 and Supplementary Table 5). The flexible MTs associated into swarms with a circular motion in the presence of $l$-DNA1, while the swarms of the rigid MTs exhibited a translational motion in the presence of $l$-DNA5 (Supplementary Movie 3). The formation of these two types of swarms was completely orthogonal, because $l$-DNA1 did not affect the rigid MTs while $l$-DNA5 did not interact with the flexible MTs (Fig. 4). The independent addressability of the MTs based on multiple logic gates will allow the design of more complex systems with diverse functionality.

Light-switched repeated swarming of MTs. To obtain a fast, reversible, and non-invasive way of altering the DNA input, we aimed to incorporate photoresponsive DNAs $(p \text {-DNAs })^{51}$. We installed a photoresponsive molecule, azobenzene, into two DNA strands which allowed the ON/OFF switching of the hybridization between these two DNA strands. The switching arises from melting temperature $\left(T_{\mathrm{m}}\right)$ changes of DNA hybridization triggered by the cis-trans isomerization of the azobenzene moiety by ultraviolet (UV) or visible light (Fig. 5a and Supplementary Table 6$)^{52}$. The two $p$-DNAs were designed such that the melting 


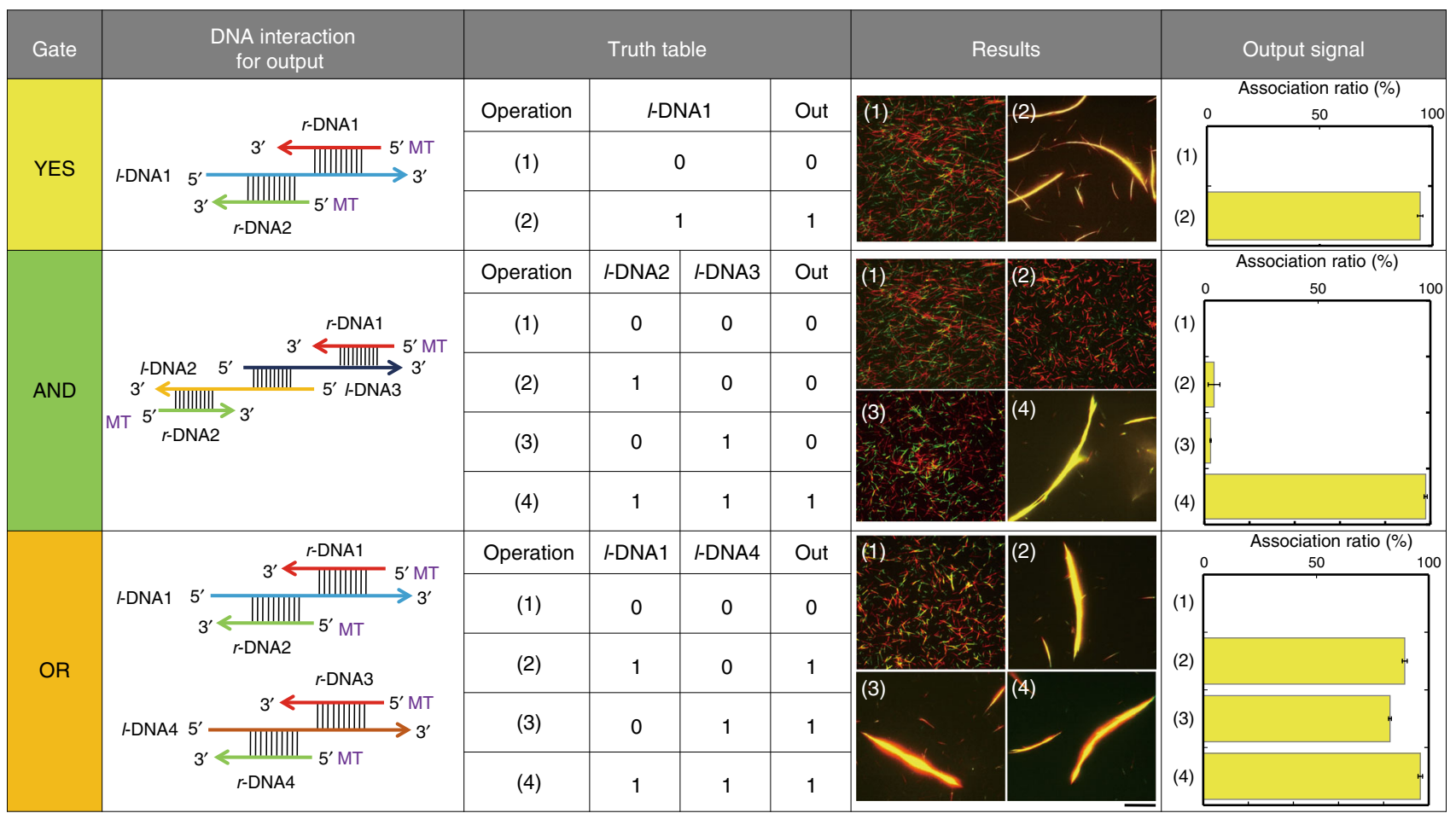

Fig. 2 Design of logic gates constructed with MTs. For the YES gate, the I-DNA1 signal was inputted into the system and swarming was obtained as the output signal (1 to 1). For the AND gate, I-DNA2 and I-DNA3 had both to be present to obtain swarming. For the OR gate, the presence of either $I$-DNA1 or I-DNA4 was sufficient to obtain swarming. The concentration of red and green MTs was $0.6 \mu \mathrm{M}$, and the conjugation ratio of each r-DNA to MTs was $\sim 100 \%$. The concentration of kinesin and each I-DNA was $0.3 \mu \mathrm{M}$ and $0.6 \mu \mathrm{M}$, respectively. Scale bar: $20 \mu \mathrm{m}$. Error bars: s.e.m.

a Rigid MTs (GMPCPP-MTs)
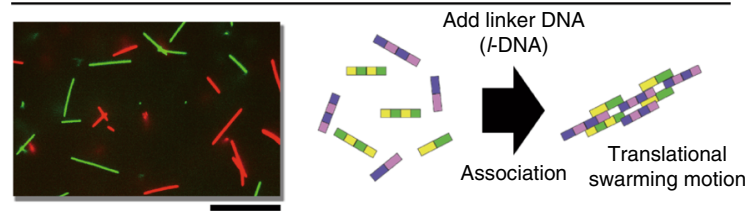

b Flexible MTs (GTP-MTs)

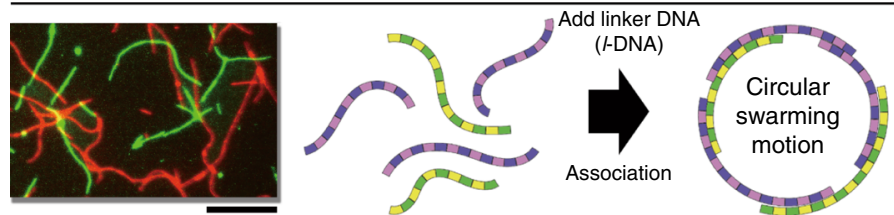

C 0
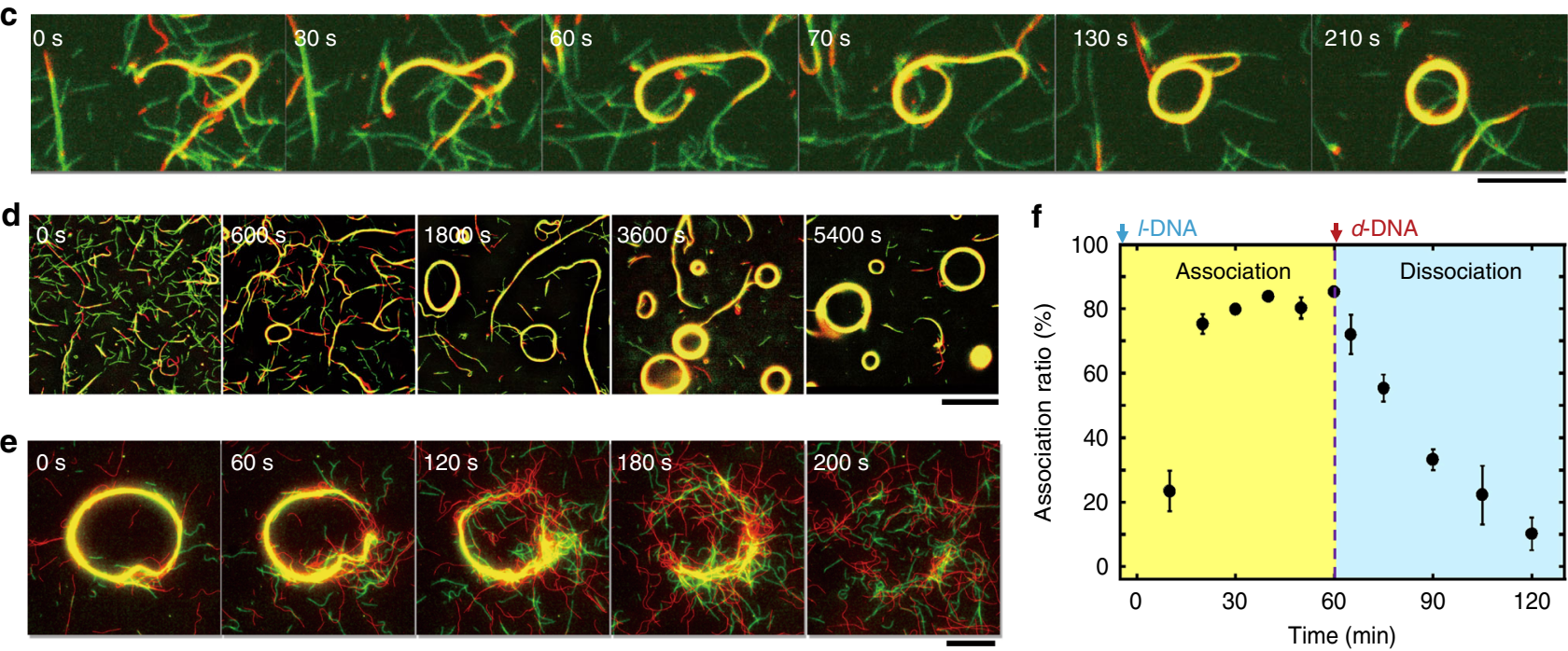

Fig. 3 Regulation of swarming mode of MTs by tuning their physical properties. a, b Fluorescence microscopy images of rigid and flexible MTs, respectively; schematic illustrations showing different swarming modes of the MTs. c Time-lapse fluorescence microscopy images showing the formation of a swarm group with a circular motion from flexible red and green MTs with lengths of $22 \pm 13 \mu \mathrm{m}$ and $16 \pm 9 \mu \mathrm{m}$ (average \pm s.d.), respectively. d Time-lapse fluorescence microscopy images showing the formation of swarms with a circular motion in the presence of $I$-DNA1 (0.6 $\mu$ M). e Dissociation of a swarm group with a circular motion triggered by the $d$-DNA input signal $(0.6 \mu \mathrm{M})$. The swarm group was made of $\sim 300$ single MTs. $\mathbf{f}$ Change of the association ratio over time upon addition of $I$-DNA1 and $d$-DNA. The concentration of the red and green MTs was $0.6 \mu M$ each, and the conjugation ratio of $r$-DNA1 or $r$-DNA2 to MTs was $\sim 100 \%$. The concentration of kinesin was $0.3 \mu \mathrm{M}$. Scale bar: $20 \mu \mathrm{m}$ (a-e). Error bar: s.e.m 


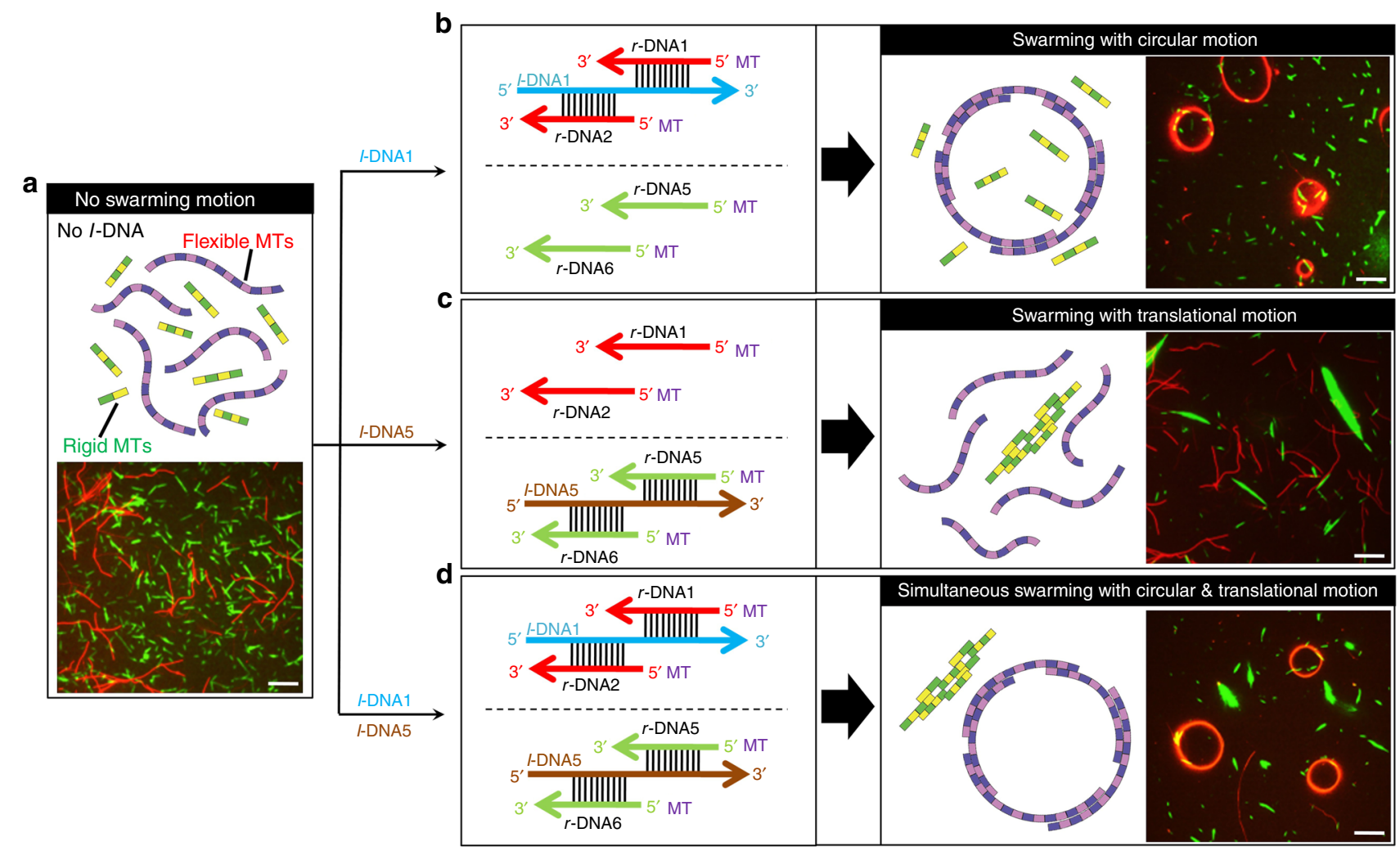

Fig. 4 Orthogonal control of swarming of MTs. a Schematic representation and fluorescence microscopy image of MTs with different rigidity. The flexible MTs (red) were conjugated with r-DNA1 and r-DNA2, while the rigid MTs (green) were conjugated with $r$-DNA5 and $r$-DNA6. b Upon inputting I-DNA1, the flexible MTs associated into circular shaped swarms through hybridization of $r$-DNA1 and $r$-DNA2 with l-DNA1 and appeared in red. c Green swarms with translational motion associated with rigid MTs were formed through hybridization of $r$-DNA5 and $r$-DNA6 with /-DNA5. d Swarms with translational and circular motions were simultaneously formed in response to the introduction of both input DNA signals. The concentration of red and green MTs was $0.6 \mu \mathrm{M}$ each, and the conjugation ratio of each $r$-DNA to MTs was $\sim 100 \%$. The concentration of kinesin was $0.3 \mu \mathrm{M}$ and that of each I-DNA was $0.6 \mu \mathrm{M}$. Scale bars: $20 \mu \mathrm{m}$

temperature is $<20^{\circ} \mathrm{C}$ in the cis state and $60^{\circ} \mathrm{C}$ in the trans state (Supplementary Fig. 5). The photoresponsive DNAs ( $p$-DNA1 and $p$-DNA2) were conjugated to MTs already fluorescently labeled with TAMRA and FAM (Fig. 5b). The functionalized MTs moved on a kinesin-coated substrate without the loss of mobility (Supplementary Fig. 6). We applied UV irradiation $(\lambda=365 \mathrm{~nm})$ to initialize the azobenzene groups to the cis form, in order to start with the movement of isolated MTs (Fig. 5c). We then irradiated the MTs with visible light $(\lambda=480 \mathrm{~nm})$, which triggered the cis to trans isomerisation of azobenzenes, permitted the hybridization of $p$-DNA1 with $p$-DNA2, and caused the swarming of MTs (Fig. 5c, rigid MTs). Subsequent irradiation with UV dissociated the swarms back into individual MTs. This lightswitched association/dissociation of MTs was repeated for three cycles (Fig. 5d). We also changed the swarming mode of photoresponsive MTs from translational to circular motion by reducing the MT rigidity (Fig. 5c, flexible MTs). Repetitive and reversible switching of the swarming of MTs with light was thus successfully achieved by installing a photoresponsive component to the system (Supplementary Movie 4).

\section{Discussion}

In response to the scientific questions, we demonstrated that DNA can be employed to selectively and reversibly control the behaviors of cytoskeletal filaments propelled by biomolecular motors and to provide sensing and information processing capabilities to these systems. Our approach complements the recently described approach to information processing where a swarm of cytoskeletal filaments propelled by biomolecular motors traverses a maze of guiding structures and in the process, computes the solution to a mathematical problem encoded in the maze ${ }^{53}$. Here, the information is not encoded in the positions of the filaments with respect to a guiding structure, but in their positions relative to each other. This work should motivate further advancement not only in chemistry, but also in bio- and DNA nanotechnology. Potential future applications are: active DNA sensors controlled by analyte oligonucleotides such as microRNA, biomimetic displays where DNA computing produces patterns, adaptive actuators designed to sense and respond to their chemical and/or physical environment, analyte concentrators which integrate preprocessing of molecular information, and sequential reactors with programmable reaction pathways. At the same time, our work capitalizes on the advantages of biomolecular motor systems, such as high energy efficiency, high specific power, and cost efficiency in handling millions of MTs, which are not yet available in conventionally fabricated mechanical swarm robots. The present work thus contributes to a new paradigm in robotics, i.e., molecular robotics.

\section{Methods}

Purification of tubulin and kinesin. Tubulin was purified from porcine brain using a high-concentration PIPES buffer (1 M PIPES, $20 \mathrm{mM}$ EGTA, and $10 \mathrm{mM}$ $\mathrm{MgCl}_{2}$ ) and stored in BRB80 buffer (80 mM PIPES, $1 \mathrm{mM} \mathrm{EGTA,} 2 \mathrm{mM} \mathrm{MgCl}$, $\mathrm{pH}$ adjusted to 6.8 using $\mathrm{KOH})^{54}$. Recombinant kinesin- 1 consisting of the first 573 amino-acid residues of human kinesin-1 was prepared as described in the 
a

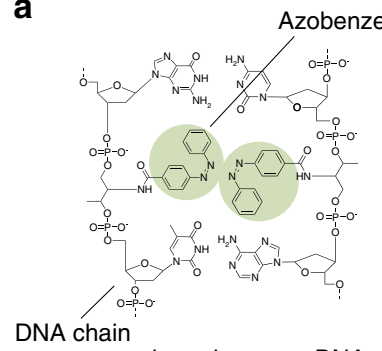

cis-azobenzene-DNA

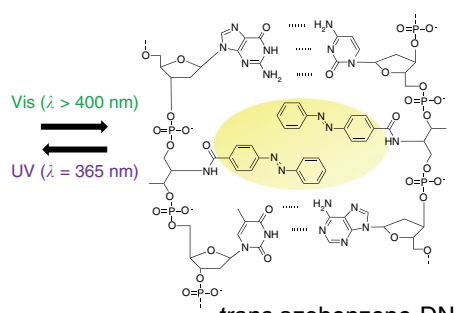

trans-azobenzene-DNA b

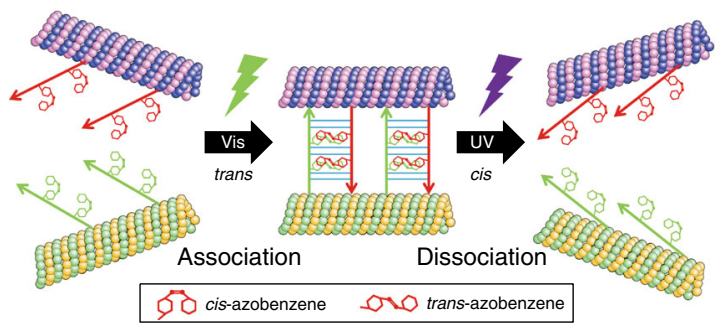

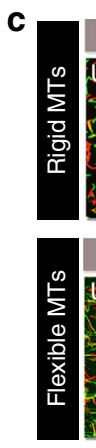
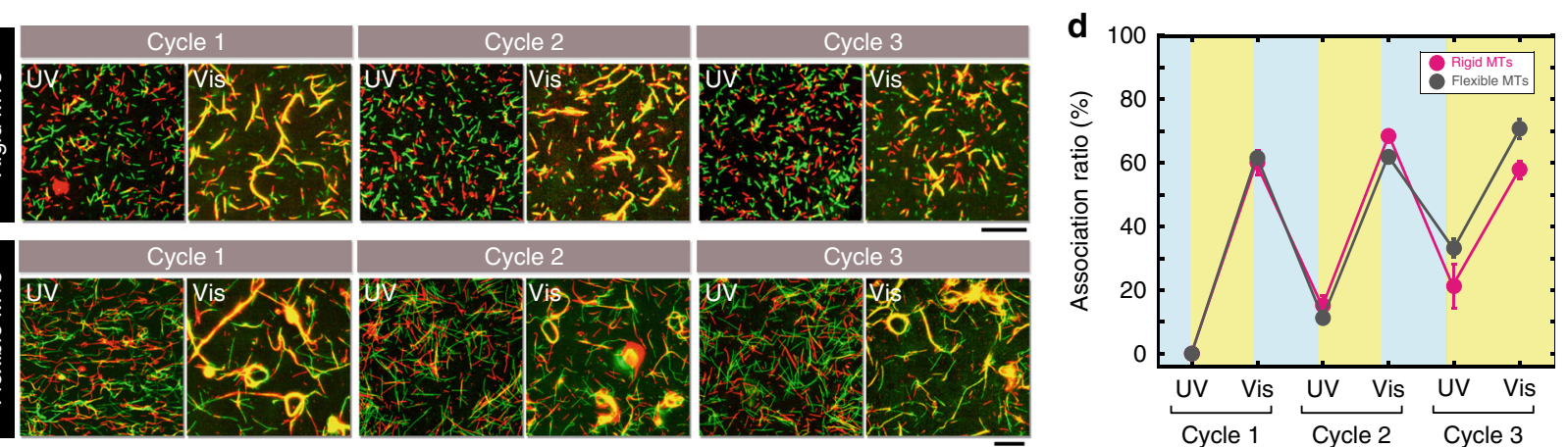

Fig. 5 Light-switched, repeated swarming of MTs. a Reversible hydrogen bonding of photoresponsive DNA ( $p$-DNA) by light-induced cis-trans isomerization of azobenzene. $\mathbf{b}$ Schematic of selective association and dissociation of $p$-DNA-conjugated MTs under UV and visible light irradiation, respectively. c Visible light $\left(\lambda=480 \mathrm{~nm}, I=1.3 \mathrm{~mW} \mathrm{~cm}^{-2}\right)$ induced isomerization of azobenzene from the cis to the trans form, which resulted in translational swarming of the $p$-DNA-conjugated rigid MTs with length of $4 \pm 2 \mu \mathrm{m}$ (average \pm s.d.). The swarms were then exposed to UV light $\left(\lambda=365 \mathrm{~nm}, I=0.4 \mathrm{~mW} \mathrm{~cm}^{-2}\right)$ for 6 min that isomerized the azobenzene from the trans to the cis form. The swarms dissociated into single MTs within $12 \mathrm{~min}$ of the onset of photoirradiation. This cycle was repeated three times. Visible light irradiation to flexible MTs with length of $12 \pm 1 \mu \mathrm{m}$ (average $\pm \mathrm{s}$.d.) generated swarms with circular motion. $\mathbf{d}$ Changes in the association ratio upon repeated irradiation by visible and UV light. The concentration of red and green MTs was $0.6 \mu \mathrm{M}$ each, and the conjugation ratio of $p$-DNA1 or p-DNA2 to MTs was $\sim 100 \%$. The concentration of kinesin was $0.8 \mu \mathrm{M}$. Scale bars: $20 \mu \mathrm{m}$. Error bar: s.e.m.

literature ${ }^{55}$. Azide labeled tubulin was prepared using $\mathrm{N}_{3}$-PEG4-NHS following the established protocol of labeling tubulin with a fluorescent dy $\mathrm{e}^{56}$. The tubulin concentration was determined by measuring the absorbance at $280 \mathrm{~nm}$ using a UV spectrophotometer (Nanodrop 2000c).

Design and preparation of DNA sequences. $r$-DNA and $l$-DNA strands were designed from $T_{\mathrm{m}}$ simulation using OligoAnalyzer 3.1 (https://sg.idtdna.com/calc/ analyzer) software with a $T_{\mathrm{m}}$ between 0 and $50^{\circ} \mathrm{C}$ for experimental testing. A further selection criterion was followed for logic gate experiments such that any undesired interactions were avoided between DNA strands (Supplementary Fig. 7). Dibenzocyclooctyne (DBCO) and fluorescent dye-labeled strands were chemically synthesized using appropriate CPG columns and a phosphoramidite (Glen Research, VA) on a ABI 3900 automatic DNA synthesizer, purified by reverse phase HPLC and fully characterized by MALDI-TOF/MS (Bruker microflex LRF). The $r$-DNA was modified at the $3^{\prime}$ end with either 5(6)-Carboxytetramethylrhodamine (TAMRA) or 5-Carboxyfluorescein (FAM) and at the $5^{\prime}$ end with DBCO. The $p$-DNA was synthesized in the laboratory according to an established protocol ${ }^{57} \cdot l$-DNA and $d$-DNA were purchased from Eurofins Genomics LLC.

Preparation of MTs. MTs were prepared by adding azide tubulin to polymerization buffer ( $80 \mathrm{mM}$ PIPES, $1 \mathrm{mM}$ EGTA, $1 \mathrm{mM} \mathrm{MgCl} 2,1 \mathrm{mM}$ polymerizing agent, $\mathrm{pH} \sim 6.8$ ) into a final concentration of $56 \mu \mathrm{M}$ tubulin incubating at $37^{\circ} \mathrm{C}$ for $30 \mathrm{~min}$. The polymerizing agent for flexible MTs was GTP, and for rigid MTs was GMPCPP, a slowly hydrolyzable analog of GTP. Dimethyl sulfoxide (DMSO) was added to a final concentration of $5 \%$, only for the polymerization of flexible MTs. Copper free click reaction was initiated by adding $3.5 \mu \mathrm{L}$ DBCO conjugated $r$ DNAs $(500 \mu \mathrm{M})$ to the $5 \mu \mathrm{L}$ azide-MTs $(56 \mu \mathrm{M})$ which allowed azide-alkyne cycloaddition reaction and incubated at $37^{\circ} \mathrm{C}$ for $6 \mathrm{~h}^{58} .100 \mu \mathrm{L}$ of cushion buffer (BRB80 buffer supplemented with $60 \%$ glycerol) was used to separate the MTs by centrifugation at $201,000 \times g\left(\mathrm{~S} 55 \mathrm{~A} 2-0156\right.$ rotor, Hitachi) for $1 \mathrm{~h}$ at $37^{\circ} \mathrm{C}$. After removing the supernatant, the pellet of $r$-DNA-conjugated MTs was washed once with $100 \mu \mathrm{L}$ BRB80P (BRB80 supplemented with $1 \mathrm{mM}$ taxol) and dissolved in 15 $\mu \mathrm{L}$ BRB80P. $p$-DNA-conjugated MTs were prepared following the same procedure.
Demonstration of swarming of MTs. A flow cell with dimensions of $9 \times 2.5 \times$ $0.45 \mathrm{~mm}^{3}(\mathrm{~L} \times \mathrm{W} \times \mathrm{H})$ was assembled from two cover glasses (MATSUNAMI Inc.) using a double-sided tape as a spacer. The flow cell was filled with $5 \mu \mathrm{L}$ casein buffer (BRB80 buffer supplemented with $0.5 \mathrm{mg} \mathrm{mL}^{-1}$ casein). After incubating for $3 \mathrm{~min}, 0.3 \mu \mathrm{M}$ kinesin solution was introduced into the flow cell and incubated for $5 \mathrm{~min}$ resulting in a kinesin density of $4000 \mu \mathrm{m}^{-2}$ on the substrate ${ }^{59}$. After washing the flow cell with $5 \mu \mathrm{L}$ of wash buffer (BRB80 buffer supplemented with $1 \mathrm{mM}$ DTT, $10 \mu \mathrm{M}$ taxol), $5 \mu \mathrm{L}$ of red MTs (TAMRA-labeled $r$-DNA MTs) solution was introduced and incubated for $2 \mathrm{~min}$, followed by washing with $10 \mu \mathrm{L}$ of wash buffer. Subsequently, $5 \mu \mathrm{L}$ of green MTs (FAM-labeled $r$-DNA MTs) solution was introduced and incubated for $2 \mathrm{~min}$, followed by washing with $10 \mu \mathrm{L}$ of motility buffer. The green MTs were incubated with $l$-DNA for $15 \mathrm{~min}$ at room temperature prior to addition to flow cell. The motility of MTs was initiated by applying $5 \mu \mathrm{L}$ ATP buffer (wash buffer supplemented with $5 \mathrm{mM} \mathrm{ATP}, 4.5 \mathrm{mg} \mathrm{mL}^{-1}$ D-glucose, $50 \mathrm{U} \mathrm{mL}^{-1}$ glucose oxidase, $50 \mathrm{U} \mathrm{mL}^{-1}$ catalase, and $0.2 \%$ methylcellulose $(\mathrm{w} / \mathrm{v})$ ). The time of ATP addition was set as $0 \mathrm{~h}$. Soon after the addition of ATP buffer, the flow cell was placed in an inert chamber system (ICS) ${ }^{60}$ and the MTs were monitored using a microscope at room temperature $\left(25^{\circ} \mathrm{C}\right)$. The experiment was performed at least 10 times for each condition.

Fluorescence microscopy. The samples were illuminated with a $100 \mathrm{~W}$ mercury lamp and visualized by an epifluorescence microscope (Eclipse Ti, Nikon) using an oil-coupled Plan Apo 60× N.A.1.4 objective (Nikon). UV cut-off filter blocks (TRITC: EX 540/25, DM565, BA605/55; GFP-B: EX460-500, DM505, BA510-560; Nikon) were used in the optical path of the microscope. Images were captured using a cooled-CMOS camera (NEO sCMOS, Andor) connected to a PC. Two ND filters (ND4, 25\% transmittance for TRITC and ND1, 100\% transmittance for GFPB) were inserted into the illumination light path of the fluorescence microscope to reduce photobleaching of the samples. In order to isomerize the azobenzene units, the flow cell was irradiated with the light passed through a UV-1A filter block (UV1A: EX 365-410, DM400, BA400; Nikon).

Data availability. The data that support this study are available from the corresponding author upon reasonable request. 
Received: 18 October 2017 Accepted: 22 December 2017

Published online: 31 January 2018

\section{References}

1. Whitesides, G. M. \& Grzybowski, B. Self-assembly at all scales. Science 295 2418-2421 (2002).

2. Bonabeau, E., Dorigo, M. \& Theraulaz, G. Swarm Intelligence: From Natural to Artificial Systems. (Oxford University Press, Oxford, New York, 1999).

3. Beshers, S. N. \& Fewell, J. H. Models of division of labor in social insects. Annu. Rev. Entomol. 46, 413-440 (2001).

4. Niven, J. E. How honeybees break a decision-making deadlock. Science $\mathbf{3 3 5}$, 43-44 (2011).

5. Seyfried, J. et al. The I-SWARM project: intelligent small world autonomous robots for micromanipulation. LNCS 3342, 70-83 (2005).

6. Turgut, A. E., Celikkanat, H., Gökçe, F. \& Sahin, E. Self-organized flocking in mobile robot swarms. Swarm Intell. 2, 97-120 (2008)

7. Wei, H. X., Chen, Y. D., Tan, J. D. \& Wang, T. M. Sambot: a self-assembly modular robot system. IEEE/ASME Trans. Mechatron. 16, 745-757 (2011).

8. Palacci, J., Sacanna, S., Steinberg, A. P., Pine, D. J. \& Chaikin, P. M. Living crystals of light-activated colloidal surfers. Science 339, 936-940 (2013).

9. $\mathrm{Xu}, \mathrm{T}$. et al. Reversible swarming and separation of self-propelled chemically powered nanomotors under acoustic fields. J. Am. Chem. Soc. 137, 2163-2166 (2015).

10. Wang, W., Duan, W., Ahmed, S., Sen, A. \& Mallouk, T. E. From one to many: dynamic assembly and collective behavior of self-propelled colloidal motors. Acc. Chem. Res. 48, 1938-1946 (2015).

11. Rubenstein, M., Cornejo, A. \& Nagpal, R. Programmable self-assembly in a thousand robot swarm. Science 345, 795-799 (2014).

12. Hagiya, M., Konagaya, A., Kobayashi, S., Saito, H. \& Murata, S. Molecular robots with sensors and intelligence. Acc. Chem. Res. 47, 1681-1690 (2014)

13. Sato, Y., Hiratsuka, Y., Kawamata, I., Murata, S. \& Nomura, S. M. Micrometer-sized molecular robot changes its shape in response to signal molecules. Sci. Robot. 2, eaal3735 (2017).

14. Kassem, S. et al. Stereodivergent synthesis with a programmable molecular machine. Nature 549, 374-378 (2017).

15. Lam, A. T. et al. Cytoskeletal motor-driven active self-assembly in in vitro systems. Soft Matter 12, 988-997 (2016).

16. Hess, H. \& Ross, J. L. Non-equilibrium assembly of MTs: from molecules to autonomous chemical robots. Chem. Soc. Rev. 46, 5570-5587 (2017).

17. Schaller, V. et al. Crosslinking proteins modulate the self-organization of driven systems. Soft Matter 9, 7229-7233 (2013).

18. Inoue, D. et al. Depletion force induced collective motion of MTs driven by kinesin. Nanoscale 7, 18054-18061 (2015).

19. Kraikivski, P., Lipowsky, R. \& Kierfeld, J. Enhanced ordering of interacting filaments by molecular motors. Phys. Rev. Lett. 96, 258103 (2006).

20. Butt, T. et al. Myosin motors drive long range alignment of actin filaments. J. Biol. Chem. 285, 4964-4974 (2010).

21. Schaller, V., Weber, C., Semmrich, C., Frey, E. \& Bausch, A. R. Polar patterns of driven filaments. Nature 467, 73-77 (2010).

22. Hess, H. et al. Molecular self-assembly of nanowires and nanospools using active transport. Nano. Lett. 5, 629-633 (2005).

23. Tamura, Y. et al. Dynamic self-organization and polymorphism of MT assembly through active interactions with kinesin. Soft Matter 7, 5654-5659 (2011).

24. Idan, O. et al. Nanoscale transport enables active self-assembly of millimeterscale wires. Nano. Lett. 12, 240-245 (2012).

25. Kumar, S. et al. Sensing protein antigen and microvesicle analytes using highcapacity biopolymer nano-carriers. Analyst 141, 836-846 (2016).

26. Sumino, Y. et al. Large-scale vortex lattice emerging from collectively moving MTs. Nature 483, 448-452 (2012).

27. Braun, M., Lansky, Z., Hilitski, F., Dogic, Z. \& Diez, S. Entropic forces drive contraction of cytoskeletal networks. BioEssays 38, 474-481 (2016).

28. Schaller, V., Weber, C., Semmrich, B., Frey, E. \& Bausch, A. R. Frozen steady states in active systems. Proc. Natl Acad. Sci. USA 108, 19183-19188 (2011).

29. Adelman, L. M. Molecular computation of solutions to combinatorial problems. Science 266, 1021-1024 (1994)

30. Qian, L. \& Winfree, E. Scaling up digital circuit computation with DNA strand displacement cascade. Science 332, 1196-1201 (2011).

31. Seeman, N. C. Nucleic-acid junctions and lattices. J. Theor. Biol. 99, 237-247 (1982).

32. Rothemund, P. W. K. Folding DNA to create nanoscale shapes and patterns. Nature 440, 297-302 (2006)
33. Rogers, A. R., Driver, J. W., Constantinou, P. E., Kenneth Jamison, D. \& Diehl, M. R. Negative interference dominates collective transport of kinesin motors in the absence of load. Phys. Chem. Chem. Phys. 11, $4882-4889$ (2009).

34. Wollman, A. J. M., Sanchez-Cano, C., Carstairs, H. M., Cross, R. A. \& Turberfield, A. J. Transport and self-organization across different length scales powered by motor proteins and programmed by DNA. Nat. Nanotechnol. 9 , 44-47 (2014).

35. Derr, N. D. et al. Tug-of-war in motor protein ensembles revealed with a programmable DNA origami scaffold. Science 338, 662-665 (2012).

36. Iwaki, M., Wickham, S. F., Ikezaki, K., Yanagida, T. \& Shih, W. M. A programmable DNA origami nanospring that reveals force-induced adjacent binding of myosin VI heads. Nat. Commun. 7, 13715 (2016).

37. Dinu, C. Z. et al. Parallel manipulation of bifunctional DNA molecules on structured surfaces using kinesin-driven MTs. Small 2, 1090-1098 (2003).

38. Diez, S. et al. Stretching and transporting DNA molecules using motor proteins. Nano. Lett. 3, 1251-1254 (2003)

39. Hiyama, S. et al. Autonomous loading, transport, and unloading of specified cargoes by using DNA hybridization and biological motor-based motility. Small 4, 410-415 (2008).

40. Hiyama, S., Gojo, R., Takeuchi, S. \& Sutoh, K. Biomolecular-motor-based nano- or microscale particle translocations on DNA microarrays. Nano. Lett. 9, 2407-2413 (2009).

41. Hiyama, S., Moritani, Y., Gojo, R., Takeuchi, S. \& Sutoh, K. Biomolecularmotor-based autonomous delivery of lipid vesicles as nano- or microscale reactors on a chip. Lab Chip 10, 2741-2748 (2010).

42. Schmidt, C. \& Vogel, V. Molecular shuttles powered by motor protein: loading and unloading stations for nanocargo integrated into one device. Lab Chip 10 2195-2198 (2010)

43. Steuerwald, D., Früh, S. M., Griss, R., Lovchik, R. D. \& Vogel, V. Nanoshuttles propelled by motor proteins sequentially assemble molecular cargo in a microfluidic device. Lab Chip 19, 3729-3738 (2014).

44. Kagan, D. et al. Functionalized micromachines for selective and rapid isolation of nucleic acid targets from complex samples. Nano. Lett. 11, 2083-2087 (2011).

45. Orozco, J. et al. Dynamic isolation and unloading of target proteins by aptamer-modified microtransporters. Anal. Chem. 83, 7962-7969 (2011).

46. Schnitzer, M. J. \& Block, S. M. Kinesin hydrolyses one ATP per 8-nm step. Nature 388, 386-390 (1997).

47. Zhang, D. Y. \& Seelig, G. Dynamic DNA nanotechnology using stranddisplacement reactions. Nat. Chem. 3, 103-111 (2011).

48. Kawamura, R., Kakugo, A., Osada, Y. \& Gong, J. P. Selective formation of a linear shaped bundle of microtubules. Langmuir 26, 533-537 (2010).

49. Wada, S. et al. Effect of length and rigidity of microtubules on the size of ring shaped assemblies obtained through active self-organization. Soft Matter 11, 1151-1157 (2015).

50. Mickey, B. \& Howard, J. Rigidity of MTs is increased by stabilizing agents. Cell Biol. 130, 909-917 (1995).

51. Asanuma, H., Liang, X., Yoshida, T. \& Komiyama, M. Photocontrol of DNA duplex formation by using azobenzene-bearing oligonucleotides. Chembiochem 2, 39-44 (2001).

52. Kamiya, Y. \& Asanuma, H. Light-driven DNA nanomachine with a photoresponsive molecular engine. Acc. Chem. Res. 47, 1663-1672 (2014).

53. Nicolau, D. V. Jr et al. Parallel computation with molecular-motor-propelled agents in nanofabricated networks. Proc. Natl Acad. Sci. USA 113, 2591-2596 (2016).

54. Castoldi, M. \& Popov, A. V. Purification of brain tubulin through two cycles of polymerization-depolymerization in a high-molarity buffer. Protein Expr. Purif. 32, 83-88 (2003)

55. Case, R. B., Pierce, D. W., Nora, H. B., Cynthia, L. H. \& Vale, R. D. The directional preference of kinesin motors is specified by an element outside of the motor catalytic domain. Cell 90, 959-966 (1997)

56. Peloquin, J., Komarova, Y. \& Borisy, G. Conjugation of fluorophores to tubulin. Nat. Methods 2, 299-303 (2005).

57. Asanuma, H. et al. Synthesis of azobenzene-tethered DNA for reversible photo-regulation of DNA functions: hybridization and transcription. Nat. Protoc. 2, 203-212 (2007)

58. Früh, S. M., Steuerwald, D., Simon, U. \& Vogel, V. Covalent cargo loading to molecular shuttles via copper-free click chemistry. Biomacromolecules 13, 3908-3911 (2012)

59. Kabir, A. M. R. et al. Buckling of MTs on a 2D elastic medium. Sci. Rep. 5, 17222 (2015)

60. Kabir, A. M. R., Inoue, D., Kakugo, A., Kamei, A. \& Gong, J. P. Prolongation of the active lifetime of a biomolecular motor for in vitro motility assay by using an inert atmosphere. Langmuir 27, 13659-13668 (2011).

\section{Acknowledgements}

We thank Prof. Akihiko Konagaya and the members of the Molecular Robotics Research Group for valuable discussions. This work was financially supported by Grant-in-Aid for Scientific Research on Innovative Areas "Molecular Robotics" (JSPS KAKENHI Grant 
Number JP24104004) from Japan Society for the Promotion of Science (JSPS) and Grantin-Aid for Challenging Exploratory Research (JSPS KAKENHI Grant Number 15K12135). H.H. was supported by NSF grant CMMI-1662329.

\section{Author contributions}

A.M.R.K., D.I., K.S., A.Ku., and A.Ka. conceived and designed the experiments. H.A. synthesized the azobenzene-amidite monomer. J.J.K. and R.S. performed the experiments, and analyzed the experimental results. J.J.K., A.M.R.K., D.I., K.S., H.H., A.Ku., and A.Ka. wrote the manuscript.

\section{Additional information}

Supplementary Information accompanies this paper at https://doi.org/10.1038/s41467017-02778-5.

Competing interests: The authors declare no competing financial interests.

Reprints and permission information is available online at http://npg.nature.com/ reprintsandpermissions/
Publisher's note: Springer Nature remains neutral with regard to jurisdictional claims in published maps and institutional affiliations.

(c) (i) Open Access This article is licensed under a Creative Commons Attribution 4.0 International License, which permits use, sharing, adaptation, distribution and reproduction in any medium or format, as long as you give appropriate credit to the original author(s) and the source, provide a link to the Creative Commons license, and indicate if changes were made. The images or other third party material in this article are included in the article's Creative Commons license, unless indicated otherwise in a credit line to the material. If material is not included in the article's Creative Commons license and your intended use is not permitted by statutory regulation or exceeds the permitted use, you will need to obtain permission directly from the copyright holder. To view a copy of this license, visit http://creativecommons.org/ licenses/by/4.0/.

(c) The Author(s) 2018 